\title{
Using sums of squares to prove that certain entire functions have only real zeros
}

\author{
by \\ George Gasper' \\ Dedicated to the memory of Ralph P. Boas, Jr. (1912-1992)
}

(May 17, 1993 version)

\begin{abstract}
It is shown how sums of squares of real valued functions can be used to give new proofs of the reality of the zeros of the Bessel functions $J_{\alpha}(z)$ when $\alpha \geq-1$, confluent hypergeometric functions ${ }_{0} F_{1}(c ; z)$ when $c>0$ or $0>c>-1$, Laguerre polynomials $L_{n}^{\alpha}(z)$ when $\alpha \geq-2$, and Jacobi polynomials $P_{n}^{(\alpha, \beta)}(z)$ when $\alpha \geq-1$ and $\beta \geq-1$. Besides yielding new inequalities for $|F(z)|^{2}$, where $F(z)$ is one of these functions, the derived identities lead to inequalities for $\partial|F(z)|^{2} / \partial y$ and $\partial^{2}|F(z)|^{2} / \partial y^{2}$, which also give new proofs of the reality of the zeros.
\end{abstract}

\section{Introduction}

In a 1975 survey paper [1] on positivity and special functions it was shown how sums of squares of special functions could be used to prove the nonnegativity of the Fejér kernel, the positivity of integrals of Bessel functions 10 and of the Cotes' numbers for some Jacobi abscissas, a Turán type inequality for Bessel functions, the Askey-Gasper inequality (cf. [1], [2], [13], [15])

$$
\sum_{k=0}^{n} P_{k}^{(\alpha, 0)}(x) \geq 0, \quad \alpha>-2, \quad-1 \leq x \leq 1,
$$

\footnotetext{
${ }^{1}$ Supported in part by the National Science Foundation under grant DMS-9103177. Key words. Entire functions, inequalities, real zeros, sums of squares, confluent hypergeometric functions, Bessel functions, Jacobi polynomials, Laguerre polynomials.
} 
which de Branges [5] employed to complete his proof of the Bieberbach conjecture, and to prove the more general inequalities [12

$$
\sum_{k=0}^{n} \frac{(\lambda+1)_{k}}{k !} \frac{(\lambda+1)_{n-k}}{(n-k) !} \frac{P_{k}^{(\alpha, \beta)}(x)}{P_{k}^{(\beta, \alpha)}(1)} \geq 0, \quad-1 \leq x \leq 1,
$$

when $0 \leq \lambda \leq \alpha+\beta$ and $\beta \geq-1 / 2$. It was also pointed out in [11] that, since one of Jensen's necessary and sufficient conditions for the Riemann Hypothesis to hold (given in Pólya [19]) is the condition that

$$
\int_{-\infty}^{\infty} \int_{-\infty}^{\infty} \Phi(s) \Phi(t) e^{i(s+t) x}(s-t)^{2 n} d s d t \geq 0, \quad-\infty<x<\infty
$$

for $n=0,1,2,3, \ldots$, where

$$
\Phi(t)=2 \sum_{k=1}^{\infty}\left(2 k^{4} \pi^{2} e^{9 t / 2}-3 k^{2} \pi e^{5 t / 2}\right) e^{-k^{2} \pi e^{2 t}}
$$

and the above integral is a square when $n=0$, the method of sums of squares is suggested for proving (1.3).

Another of Jensen's necessary and sufficient conditions for the Riemann Hypothesis to hold is that

$$
\int_{-\infty}^{\infty} \int_{-\infty}^{\infty} \Phi(s) \Phi(t) e^{i(s+t) x} e^{(s-t) y}(s-t)^{2} d s d t \geq 0, \quad-\infty<x, y<\infty,
$$

which can also be written in the equivalent form

$$
\frac{\partial^{2}}{\partial y^{2}}|\Xi(x+i y)|^{2} \geq 0, \quad-\infty<x, y<\infty
$$

with

$$
\Xi(z)=\int_{-\infty}^{\infty} \Phi(t) \exp (i z t) d t=2 \int_{0}^{\infty} \Phi(t) \cos (z t) d t
$$

That (1.6) is a sufficient condition for the Riemann $\Xi(z)$ function to have only real zeros follows directly from observation that, since $|\Xi(x+i y)|^{2}=\Xi(x+$ $i y) \Xi(x-i y)$ is a nonnegative even function of $\mathrm{y},(1.6)$ implies that $|\Xi(x+i y)|^{2}$ 
is a nonnegative even convex function of $y$ with its unique minimum at $y=0$, and hence $\Xi(x+i y) \neq 0$ whenever $y \neq 0$. If the function $\Phi(t)$ in $(1.3)$ and (1.5) is replaced by a function $\Psi(t)$ such that the conditions stated in [19, $\S 1]$, are satisfied, then, by [19, pp. 17, 18], the inequalities in (1.3) and (1.5) are necessary and sufficient conditions for the Fourier (or cosine) transform of $\Psi(t)$ to have only real zeros. In 1913 Jensen [18] proved that each of the inequalities

$$
y \frac{\partial}{\partial y}|F(x+i y)|^{2} \geq 0, \quad \frac{\partial^{2}}{\partial y^{2}}|F(x+i y)|^{2} \geq 0, \quad-\infty<x, y<\infty,
$$

is necessary and sufficient for a real entire function $F(z) \not \equiv 0$ of genus 0 or 1 (cf. Boas [4, Chapter 2]) to have only real zeros. Also see Titchmarsh [21] and Varga [22, Chapter 3].

In view of these observations and the successes of the sums of squares method (also see [14], [16, Chapter 8]), since the early 1970's I have been investigating how squares of real valued functions can be used to prove that certain entire functions have only real zeros and to prove inequalities of the form in (1.8). In this paper I demonstrate how certain series expansions in sums of squares of special functions give new proofs of the reality of the zeros of the Bessel functions $J_{\alpha}(z)$ when $\alpha \geq-1$, confluent hypergeometric functions ${ }_{0} F_{1}(c ; z)$ when $c>0$ or $0>c>-1$, Laguerre polynomials $L_{n}^{\alpha}(z)$ when $\alpha \geq-2$, and Jacobi polynomials $P_{n}^{(\alpha, \beta)}(z)$ when $\alpha \geq-1$ and $\beta \geq-1$. Here, as elsewhere, $z=x+i y$ is a complex variable and $x$ and $y$ are real variables. For the definitions of these functions and their properties, see Erdélyi [9] and Szegö [20]. In addition, it will be shown that besides yielding new inequalities for $|F(z)|^{2}$, where $F(z)$ is one of these functions, the derived identities lead to inequalities for $\partial|F(z)|^{2} / \partial y$ and $\partial^{2}|F(z)|^{2} / \partial y^{2}$, which also give new proofs of the reality of the zeros.

\section{Initial observations}

In order to see how easily sums of squares can be used to prove that all of the zeros of $\sin z$ and $\cos z$ are real, it suffices to observe that we have the (easily verified) identities

$$
|\sin z|^{2}=\sin ^{2} x+\sinh ^{2} y
$$




$$
|\cos z|^{2}=\cos ^{2} x+\sinh ^{2} y
$$

and to note that $\sinh y=\left(e^{y}-e^{-y}\right) / 2>0$ when $y>0$, and $\sinh y<0$ when $y<0$.

One can also take partial derivatives of the identities in (2.1) and (2.2) with respect to $y$ to obtain

$$
\frac{\partial}{\partial y}|\sin z|^{2}=\frac{\partial}{\partial y}|\cos z|^{2}=\sinh 2 y
$$

which shows that $|\sin z|^{2}$ and $|\cos z|^{2}$ are increasing (decreasing) functions of $y$ when $y>0(y<0)$, and to obtain

$$
\frac{\partial^{2}}{\partial y^{2}}|\sin z|^{2}=\frac{\partial^{2}}{\partial y^{2}}|\cos z|^{2}=2 \cosh 2 y=2\left(\cosh ^{2} y+\sinh ^{2} y\right) \geq 2,
$$

which shows that $|\sin z|^{2}$ and $|\cos z|^{2}$ are convex functions of $y$. Then, because $|\sin z|^{2}$ and $|\cos z|^{2}$ are nonnegative even functions of $y$, it immediately follows from (2.3) and (2.4) that $\sin z$ and $\cos z$ have only real zeros.

Observe that the reality of the zeros of $\sin z$ and $\cos z$ also follows from the inequalities

$$
\begin{gathered}
|\sin z|^{2}>\sin ^{2} x, \quad|\cos z|^{2}>\cos ^{2} x, \quad(y \neq 0) \\
|\sin z|^{2} \geq \sinh ^{2} y, \quad|\cos z|^{2} \geq \sinh ^{2} y \\
y \frac{\partial}{\partial y}|\sin z|^{2}=y \frac{\partial}{\partial y}|\cos z|^{2} \geq 2 y^{2} \\
\frac{\partial^{2}}{\partial y^{2}}|\sin z|^{2}=\frac{\partial^{2}}{\partial y^{2}}|\cos z|^{2} \geq 2 \cosh ^{2} y \\
\frac{\partial^{2}}{\partial y^{2}}|\sin z|^{2}=\frac{\partial^{2}}{\partial y^{2}}|\cos z|^{2} \geq 2+2 \sinh ^{2} y
\end{gathered}
$$

which are consequences of (2.1)-(2.4). 


\section{Bessel functions and ${ }_{0} F_{1}(c ; z)$ functions}

Since the identities and inequalities in $\S 2$ give the reality of the zeros of the Bessel functions [20, (1.71.2)]

$$
J_{-\frac{1}{2}}(z)=\left(\frac{2}{\pi z}\right)^{\frac{1}{2}} \cos z, \quad J_{\frac{1}{2}}(z)=\left(\frac{2}{\pi z}\right)^{\frac{1}{2}} \sin z,
$$

this suggests that it should be possible to use sums of squares to prove Lommel's theorem (see Watson [23, p. 482]) that all of the zeros of the Bessel function [9, 7.2(3)]

$$
J_{\alpha}(z)=\frac{(z / 2)^{\alpha}}{\Gamma(\alpha+1)}{ }_{0} F_{1}\left(\alpha+1 ;-z^{2} / 4\right)
$$

are real when $\alpha>-1$. With this aim in mind and in order to work with entire functions, we set

$$
\mathcal{J}_{\alpha}(z)=z^{-\alpha} J_{\alpha}(z)=\frac{2^{-\alpha}}{\Gamma(\alpha+1)}{ }_{0} F_{1}\left(\alpha+1 ;-z^{2} / 4\right),
$$

which is an even entire function of $z$ such that $\overline{\mathcal{J}_{\alpha}(z)}=\mathcal{J}_{\alpha}(\bar{z})$ when $\alpha$ is real.

Let $\alpha>-1$. Then, from the product formula (37) in Carlitz [8],

$$
\left|\mathcal{J}_{\alpha}(z)\right|^{2}=\sum_{k=0}^{\infty} \frac{\left(\alpha+\frac{1}{2}\right)_{k} 2^{k-\alpha}}{k !(2 \alpha+1)_{k} \Gamma(\alpha+1)}\left(x^{2}+y^{2}\right)^{k} \mathcal{J}_{\alpha+k}(2 x) .
$$

To express each of the Bessel functions on the right side of (3.4) as a sum of squares of real valued Bessel functions observe that from the addition theorem for Bessel functions [9, 7.15(30)] we have the expansion

$$
\mathcal{J}_{\alpha+k}(2 x)=2^{k+\alpha} \Gamma(k+\alpha) \sum_{j=0}^{\infty} \frac{(j+k+\alpha)(2 k+2 \alpha)_{j}}{j !}(-1)^{j} x^{2 j}\left(\mathcal{J}_{\alpha+j+k}(x)\right)^{2} .
$$

Hence, substituting (3.5) into (3.4) and changing the order of summation we find that

$$
\begin{aligned}
\left|\mathcal{J}_{\alpha}(z)\right|^{2}=\sum_{n=0}^{\infty} \frac{(n+\alpha)(2 \alpha)_{n}}{\alpha n !}(-1)^{n} x^{2 n} & \\
& \quad \times{ }_{2} F_{1}\left(-n, n+2 \alpha ; 2 \alpha+1 ; 1+y^{2} / x^{2}\right)\left(\mathcal{J}_{\alpha+n}(x)\right)^{2}
\end{aligned}
$$


Now apply the Euler transformation formula [9, 2.9(3)]

$$
{ }_{2} F_{1}(a, b ; c ; z)=(1-z)^{-a}{ }_{2} F_{1}(a, c-b ; c ; z /(z-1))
$$

to the above ${ }_{2} F_{1}$ series to obtain the desired sum of squares expansion formula

$$
\begin{aligned}
\left|\mathcal{J}_{\alpha}(z)\right|^{2} & =\left(\mathcal{J}_{\alpha}(x)\right)^{2}+2(\alpha+1) y^{2}\left(\mathcal{J}_{\alpha+1}(x)\right)^{2} \\
& +\sum_{n=2}^{\infty} \frac{(2 n+2 \alpha)(2 \alpha+1)_{n-1}}{n !} y^{2 n} \\
& \times{ }_{2} F_{1}\left(-n, 1-n ; 2 \alpha+1 ; 1+x^{2} / y^{2}\right)\left(\mathcal{J}_{\alpha+n}(x)\right)^{2} .
\end{aligned}
$$

When $n \geq 2, \alpha>-1$ and $y \neq 0$, the positivity of the coefficients of $\left(\mathcal{J}_{\alpha+n}(x)\right)^{2}$ in $(3.8)$ follows from

$$
\begin{aligned}
(2 \alpha+1){ }_{2} F_{1}( & \left.-n, 1-n ; 2 \alpha+1 ; 1+x^{2} / y^{2}\right)=\left(2 \alpha+1+n^{2}-n\right) \\
& +n(n-1) x^{2} / y^{2}+\sum_{k=2}^{n} \frac{(-n)_{k}(1-n)_{k}}{k !(2 \alpha+2)_{k-1}}\left(1+x^{2} / y^{2}\right)^{k}>0 .
\end{aligned}
$$

Hence, since the real zeros of $\mathcal{J}_{\alpha}(x)$ and $\mathcal{J}_{\alpha+1}(x)$ are interlaced, (3.8) gives a sum of squares proof that the entire functions $\mathcal{J}_{\alpha}(z)$, and thus the Bessel functions $J_{\alpha}(z)$, have only real zeros when $\alpha>-1$. Letting $\alpha \rightarrow-1$ it follows that the Bessel function $\mathcal{J}_{-1}(z)=\lim _{\alpha \rightarrow-1} \mathcal{J}_{\alpha}(z)=-\mathcal{J}_{1}(z)$ has only real zeros.

Notice that the inequality

$$
\left|\mathcal{J}_{\alpha}(z)\right|^{2} \geq\left(\mathcal{J}_{\alpha}(x)\right)^{2}+2(\alpha+1)\left(y \mathcal{J}_{\alpha+1}(x)\right)^{2}>0, y \neq 0, \alpha>-1,
$$

and in fact infinitely many inequalities follow from (3.8) by just dropping terms from the right side of (3.8). Analogous to (2.7)-(2.9), it follows by differentiating equation (3.6) with respect to $y$ and applying (3.7) that we also have the identities

$$
\begin{aligned}
y \frac{\partial}{\partial y}\left|\mathcal{J}_{\alpha}(z)\right|^{2}=4 y^{2} \sum_{n=0}^{\infty} & \frac{(n+\alpha+1)(2 \alpha+2)_{n}}{n !} y^{2 n} \\
& \times{ }_{2} F_{1}\left(-n,-n ; 2 \alpha+2 ; 1+x^{2} / y^{2}\right)\left(\mathcal{J}_{\alpha+n+1}(x)\right)^{2}
\end{aligned}
$$


and

$$
\begin{aligned}
\frac{\partial^{2}}{\partial y^{2}}\left|\mathcal{J}_{\alpha}(z)\right|^{2} & =4 \sum_{n=0}^{\infty} \frac{(n+\alpha+1)(2 \alpha+2)_{n}}{n !} y^{2 n} \\
& \times{ }_{2} F_{1}\left(-n,-n ; 2 \alpha+2 ; 1+x^{2} / y^{2}\right)\left(\mathcal{J}_{\alpha+n+1}(x)\right)^{2} \\
& +8 y^{2} \sum_{n=0}^{\infty} \frac{(n+\alpha+2)(2 \alpha+3)_{n+1}}{n !} y^{2 n} \\
& \times{ }_{2} F_{1}\left(-n,-n-1 ; 2 \alpha+3 ; 1+x^{2} / y^{2}\right)\left(\mathcal{J}_{\alpha+n+2}(x)\right)^{2}
\end{aligned}
$$

which give infinitely many inequalities, such as, e.g.,

$$
\begin{gathered}
y \frac{\partial}{\partial y}\left|\mathcal{J}_{\alpha}(z)\right|^{2} \geq 4(\alpha+1)\left(y \mathcal{J}_{\alpha+1}(x)\right)^{2} \geq 0, \quad \alpha \geq-1, \\
\frac{\partial^{2}}{\partial y^{2}}\left|\mathcal{J}_{\alpha}(z)\right|^{2} \geq 4(\alpha+1)\left(\mathcal{J}_{\alpha+1}(x)\right)^{2} \geq 0, \quad \alpha \geq-1,
\end{gathered}
$$

each of which proves that $J_{\alpha}(z)$ has only real zeros when $\alpha \geq-1$.

In view of (3.3) the reality of the zeros of $\mathcal{J}_{\alpha}(z)$ when $\alpha>-1$ is equivalent to the statement that all of the zeros of the confluent hypergeometric function ${ }_{0} F_{1}(c ; z)$ are real and negative when $c>0$. However, it is known [17 that the zeros of ${ }_{0} F_{1}(c ; z)$ are also real (but not necessarily negative) when $-1<$ $c<0$. Because this fact does not follow from (3.8) or (3.11)-(3.14), we will next show how it can also be proved by using sums of squares of real value functions.

From formulas (53) and (52) in Burchnall and Chaundy [7] it follows that if $c$ is real valued and $c \neq 0,-1,-2, \ldots$, then we have the expansion formulas

$$
\left|{ }_{0} F_{1}(c ; z)\right|^{2}=\sum_{k=0}^{\infty} \frac{1}{k !(c)_{k}(c)_{2 k}}\left(x^{2}+y^{2}\right)^{k}{ }_{0} F_{1}(c+2 k ; 2 x)
$$

and

${ }_{0} F_{1}(c+2 k ; 2 x)=\sum_{j=0}^{\infty} \frac{(-1)^{j}}{j !(c+2 k+j-1)_{j}(c+2 k)_{2 j}} x^{2 j}\left({ }_{0} F_{1}(c+2 k+2 j ; x)\right)^{2}$. 
As in the Bessel function case, substitute (3.16) into (3.15) and change the order of summation to get

$$
\begin{aligned}
\left|{ }_{0} F_{1}(c ; z)\right|^{2}= & \sum_{n=0}^{\infty} \\
& \frac{(-1)^{n}}{n !(c+n-1)_{n}(c)_{2 n}} x^{2 n} \\
& \times{ }_{2} F_{1}\left(-n, n+c-1 ; c ; 1+y^{2} / x^{2}\right)\left({ }_{0} F_{1}(c+2 n ; x)\right)^{2}
\end{aligned}
$$

which, by applying the transformation formula (3.7), gives

$$
\begin{aligned}
\left|{ }_{0} F_{1}(c ; z)\right|^{2}=\sum_{n=0}^{\infty} & \frac{1}{n !(n+c-1)_{n}(c)_{2 n}} y^{2 n} \\
& \times{ }_{2} F_{1}\left(-n, 1-n ; c ; 1+x^{2} / y^{2}\right)\left({ }_{0} F_{1}(c+2 n ; x)\right)^{2} .
\end{aligned}
$$

When $c>0$ and $y \neq 0$ the coefficient of $\left({ }_{0} F_{1}(c+2 n ; x)\right)^{2}$ in the series in (3.18) is obviously positive. Hence, since ${ }_{0} F_{1}(c ; x)>0$ when $c>0$ and $x \geq 0$, (3.18) gives another proof that ${ }_{0} F_{1}(c ; z)$ has only real negative zeros when $c>0$.

To handle the case $-1<c<0$ differentiate equation (3.17) with respect to $y$ and apply (3.7) to obtain

$$
\begin{aligned}
& y \frac{\partial}{\partial y}\left|c_{0} F_{1}(c ; z)\right|^{2}=2 y^{2} \sum_{n=0}^{\infty} \frac{(c+1)_{n}}{n !(c+1)_{2 n}(c+1)_{2 n+1}} y^{2 n} \\
& \times{ }_{2} F_{1}\left(-n,-n ; c+1 ; 1+x^{2} / y^{2}\right)\left({ }_{0} F_{1}(c+2 n+2 ; x)\right)^{2}
\end{aligned}
$$

and

$$
\begin{aligned}
\frac{\partial^{2}}{\partial y^{2}}\left|c_{0} F_{1}(c ; z)\right|^{2} & =2 \sum_{n=0}^{\infty} \frac{(c+1)_{n}}{n !(c+1)_{2 n}(c+1)_{2 n+1}} y^{2 n} \\
& \times{ }_{2} F_{1}\left(-n,-n ; c+1 ; 1+x^{2} / y^{2}\right)\left({ }_{0} F_{1}(c+2 n+2 ; x)\right)^{2} \\
& +4 y^{2} \sum_{n=0}^{\infty} \frac{(c+2)_{n+1}}{n !(c+1)_{2 n+2}(c+1)_{2 n+3}} y^{2 n} \\
& \times{ }_{2} F_{1}\left(-n,-n-1 ; c+2 ; 1+x^{2} / y^{2}\right)\left({ }_{0} F_{1}(c+2 n+4 ; x)\right)^{2}
\end{aligned}
$$

which, in particular, give the inequalities

$$
y \frac{\partial}{\partial y}\left|c(c+1){ }_{0} F_{1}(c ; z)\right|^{2} \geq 2(c+1)\left(y_{0} F_{1}(c+2 ; x)\right)^{2}, \quad c \geq-1,
$$


and

$$
\frac{\partial^{2}}{\partial y^{2}}\left|c(c+1){ }_{0} F_{1}(c ; z)\right|^{2} \geq 2(c+1)\left({ }_{0} F_{1}(c+2 ; x)\right)^{2}, \quad c \geq-1 .
$$

Since the coefficients on the right hand sides of (3.19)-(3.22) are clearly positive when $c>-1$ and $y \neq 0$, these formulas prove that the functions $c(c+1){ }_{0} F_{1}(c ; z)$ have only real zeros when $c \geq-1$, where it is understood that $c(c+1){ }_{0} F_{1}(c ; z)$ is to be replaced by its $c \rightarrow 0$ limit case $z_{0} F_{1}(2 ; z)$ when $c=0$, and by its $c \rightarrow-1$ limit case $z^{2}{ }_{0} F_{1}(3 ; z) / 2$ when $c=-1$.

\section{Laguerre polynomials and ${ }_{1} F_{1}(a ; c ; z)$ func- tions}

When $\alpha>-1$ the Laguerre polynomials

$$
L_{n}^{\alpha}(z)=\frac{(\alpha+1)_{n}}{n !}{ }_{1} F_{1}(-n ; \alpha+1 ; z)
$$

satisfy the orthogonality relation

$$
\int_{0}^{\infty} L_{n}^{\alpha}(x) L_{m}^{\alpha}(x) x^{\alpha} e^{-x} d x=\frac{\Gamma(n+\alpha+1)}{n !} \delta_{n m}, \quad n, m=0,1,2, \ldots,
$$

from which it follows by a standard argument (cf. [20, §3.3]) that the zeros of $L_{n}^{\alpha}(z)$ are real and positive. Analogous to the last part of the previous section, in this section we will derive some sums of squares expansions which, besides proving the reality of the zeros of these polynomials when $\alpha>-1$, also prove that they have only real zeros (not necessarily positive) when $-1 \geq \alpha \geq-2$, where $L_{n}^{\alpha}(z)$ is defined to be the $\alpha \rightarrow-k$ limit case of (4.1) when $\alpha$ is a negative integer $-k$. Thus $L_{1}^{\alpha}(z)=\alpha+1-z$, which has a negative zero when $\alpha<-1$, and $L_{2}^{\alpha}(z)=\left((\alpha+1)(\alpha+2)-2(\alpha+2) z+z^{2}\right) / 2$, which has non-real zeros when $\alpha<-2$. 
Let $\alpha$ be real valued. Substituting the sum of squares of Laguerre polynomials expansion (from [7, (91)])

$$
\begin{aligned}
L_{n-k}^{\alpha+2 k}(2 x) & =\sum_{j=0}^{n-k} \frac{(n-k-j) !(2 k+2 j+\alpha)(2 k+\alpha)_{j}}{j !(2 k+\alpha)(2 k+\alpha+1)_{n+j-k}} \\
& \times(-1)^{j} x^{2 j}\left(L_{n-k-j}^{\alpha+2 k+2 j}(x)\right)^{2}
\end{aligned}
$$

into the special case of [3, (5.4)]

$$
\left|L_{n}^{\alpha}(z)\right|^{2}=\frac{(\alpha+1)_{n}}{n !} \sum_{k=0}^{n} \frac{1}{k !(\alpha+1)_{k}}\left(x^{2}+y^{2}\right)^{k} L_{n-k}^{\alpha+2 k}(2 x)
$$

and changing the order of summation yields

$$
\begin{aligned}
\left|L_{n}^{\alpha}(z)\right|^{2}=\frac{(\alpha+1)_{n}}{n !} & \sum_{k=0}^{n} \frac{(n-k) !(2 k+\alpha)(\alpha)_{k}}{k ! \alpha(\alpha+1)_{n+k}}(-1)^{k} x^{2 k} \\
& \times{ }_{2} F_{1}\left(-k, k+\alpha ; \alpha+1 ; 1+y^{2} / x^{2}\right)\left(L_{n-k}^{\alpha+2 k}(x)\right)^{2} .
\end{aligned}
$$

Then application of (3.7) gives

$$
\begin{aligned}
\left|L_{n}^{\alpha}(z)\right|^{2}=\frac{(\alpha+1)_{n}}{n !} & \sum_{k=0}^{n} \frac{(n-k) !(2 k+\alpha)(\alpha)_{k}}{k ! \alpha(\alpha+1)_{n+k}} y^{2 k} \\
& \times{ }_{2} F_{1}\left(-k, 1-k ; \alpha+1 ; 1+x^{2} / y^{2}\right)\left(L_{n-k}^{\alpha+2 k}(x)\right)^{2}
\end{aligned}
$$

Since $L_{0}^{\alpha}(x) \equiv 1$ and the coefficients on the right hand side of (4.6) are clearly positive when $\alpha>-1$ and $y \neq 0$, the expansion (4.6) proves that the Laguerre polynomials have only real zeros when $\alpha>-1$. This also follows, in particular, from the inequalities

$$
\left|L_{n}^{\alpha}(z)\right|^{2} \geq \frac{(\alpha+1)_{n}}{n ! n !(n+\alpha)_{n}} y_{2}^{2 n} F_{1}\left(-n, 1-n ; \alpha+1 ; 1+x^{2} / y^{2}\right), \quad \alpha>-1,
$$

and

$$
\left|L_{n}^{\alpha}(z)\right|^{2} \geq\left|L_{n}^{\alpha}(x)\right|^{2}+\frac{(\alpha+1)_{n}}{n ! n !(n+\alpha)_{n}} y^{2 n}, \quad \alpha>-1, n \geq 1,
$$


which are consequences of (4.6).

Now differentiate equation (4.5) with respect to $y$ and apply (3.7) to derive the expansions

$$
\begin{aligned}
y \frac{\partial}{\partial y}\left|L_{n}^{\alpha}(z)\right|^{2} & =2 y^{2} \sum_{k=0}^{n-1} \frac{(n-k-1) !(2 k+\alpha+2)(\alpha+2)_{k}}{n ! k !(n+\alpha+1)_{k+1}} y^{2 k} \\
& \times{ }_{2} F_{1}\left(-k,-k ; \alpha+2 ; 1+x^{2} / y^{2}\right)\left(L_{n-k-1}^{\alpha+2 k+2}(x)\right)^{2}, \quad n \geq 1
\end{aligned}
$$

and

$$
\begin{aligned}
\frac{\partial^{2}}{\partial y^{2}}\left|L_{n}^{\alpha}(z)\right|^{2} & =2 \sum_{k=0}^{n-1} \frac{(n-k-1) !(2 k+\alpha+2)(\alpha+2)_{k}}{n ! k !(n+\alpha+1)_{k+1}} y^{2 k} \\
& \times{ }_{2} F_{1}\left(-k,-k ; \alpha+2 ; 1+x^{2} / y^{2}\right)\left(L_{n-k-1}^{\alpha+2 k+2}(x)\right)^{2} \\
& +4 y^{2} \sum_{k=0}^{n-2} \frac{(n-k-2) !(2 k+\alpha+4)(\alpha+3)_{k+1}}{n ! k !(n+\alpha+1)_{k+2}} y^{2 k} \\
& \times{ }_{2} F_{1}\left(-k,-k-1 ; \alpha+3 ; 1+x^{2} / y^{2}\right)\left(L_{n-k-2}^{\alpha+2 k+4}(x)\right)^{2}, \quad n \geq 1,
\end{aligned}
$$

which yield, e.g., the inequalities

$$
y \frac{\partial}{\partial y}\left|L_{n}^{\alpha}(z)\right|^{2} \geq \frac{2(\alpha+2)}{n(n+\alpha+1)}\left(y L_{n-1}^{\alpha+2}(x)\right)^{2}, \quad \alpha>-2, n \geq 1,
$$

and

$$
\frac{\partial^{2}}{\partial y^{2}}\left|L_{n}^{\alpha}(z)\right|^{2} \geq \frac{2(\alpha+2)}{n(n+\alpha+1)}\left(L_{n-1}^{\alpha+2}(x)\right)^{2}, \quad \alpha>-2, n \geq 1,
$$

and prove (after letting $\alpha \rightarrow-2$ ) that the polynomials $L_{n}^{\alpha}(z)$ have only real zeros when $\alpha \geq-2$.

For the confluent hypergeometric functions ${ }_{1} F_{1}(a ; c ; z)$ with $a$ and $c$ real valued and $c \neq 0,-1,-2, \ldots$, use of the expansion formulas $[7,(42)$ and (43)] instead of (4.3) and (4.4) yields the nonterminating extension of (4.5)

$$
\begin{aligned}
\left|{ }_{1} F_{1}(a ; c ; z)\right|^{2} & =\sum_{k=0}^{\infty} \frac{(a)_{k}(c-a)_{k}}{k !(c)_{2 k}(c+k-1)_{k}} x^{2 k} \\
& \times{ }_{2} F_{1}\left(-k, c+k-1 ; c ; 1+y^{2} / x^{2}\right)\left({ }_{1} F_{1}(a+k ; c+2 k ; x)\right)^{2}
\end{aligned}
$$


and hence, by (3.7),

$$
\begin{aligned}
\left|{ }_{1} F_{1}(a ; c ; z)\right|^{2} & =\sum_{k=0}^{\infty} \frac{(a)_{k}(c-a)_{k}}{k !(c)_{2 k}(c+k-1)_{k}}(-1)^{k} y^{2 k} \\
& \times{ }_{2} F_{1}\left(-k, 1-k ; c ; 1+x^{2} / y^{2}\right)\left({ }_{1} F_{1}(a+k ; c+2 k ; x)\right)^{2}
\end{aligned}
$$

Then differentiation of equation (4.13) with respect of $y$ and application of (3.7) gives the following extensions of (4.9) and (4.10) (and also of (3.19) and $(3.20))$, respectively,

$$
\begin{aligned}
y \frac{\partial}{\partial y} \mid c(c+ & 1)\left._{1} F_{1}(a ; c ; z)\right|^{2}=2 y^{2} \sum_{k=0}^{\infty} \frac{(a)_{k+1}(c-a)_{k+1}(c+1)}{k !(c+2)_{2 k}(c+k+1)_{k}}(-1)^{k+1} y^{2 k} \\
& \times{ }_{2} F_{1}\left(-k,-k ; c+1 ; 1+x^{2} / y^{2}\right)\left({ }_{1} F_{1}(a+k+1 ; c+2 k+2 ; x)\right)^{2}
\end{aligned}
$$

and

$$
\begin{aligned}
\frac{\partial^{2}}{\partial y^{2}} & \left|c(c+1){ }_{1} F_{1}(a ; c ; z)\right|^{2}=2 \sum_{k=0}^{\infty} \frac{(a)_{k+1}(c-a)_{k+1}(c+1)}{k !(c+2)_{2 k}(c+k+1)_{k}}(-1)^{k+1} y^{2 k} \\
& \times{ }_{2} F_{1}\left(-k,-k ; c+1 ; 1+x^{2} / y^{2}\right)\left({ }_{1} F_{1}(a+k+1 ; c+2 k+2 ; x)\right)^{2} \\
& +4 y^{2} \sum_{k=0}^{\infty} \frac{(a)_{k+2}(c-a)_{k+2}}{k !(c+2)_{2 k+2}(c+k+3)_{k}}(-1)^{k} y^{2 k} \\
& \times{ }_{2} F_{1}\left(-k,-k-1 ; c+2 ; 1+x^{2} / y^{2}\right)\left({ }_{1} F_{1}(a+k+2 ; c+2 k+4 ; x)\right)^{2} .
\end{aligned}
$$

If $a=-n$ is a negative integer and $c=\alpha+1$, then (4.13)-(4.16) reduce to (4.5), (4.6), (4.9), (4.10), respectively. If $a=c+n$ with $n$ a nonnegative integer, then (4.15) and (4.16) reduce to terminating sums of squares expansions with nonnegative coefficients which prove that $c(c+1){ }_{1} F_{1}(c+n ; c ; z)$, as a function of $z$, has only real zeros when $c \geq-1$, where this function is to be replaced by its $c \rightarrow 0$ and $c \rightarrow-1$ limit cases when $c=0$ and $c=-1$, respectively. It should be noted that, in view of Kummer's transformation formula [9, 6.3(7)]

$$
{ }_{1} F_{1}(a ; c ; x)=e^{x}{ }_{1} F_{1}(c-a ; c ;-x),
$$

these results on the zeros of $c(c+1){ }_{1} F_{1}(c+n ; c ; z)$ are equivalent to those obtained above for the Laguerre polynomials. 


\section{Jacobi polynomials}

When $\alpha>-1$ and $\beta>-1$ the Jacobi polynomials

$$
P_{n}^{(\alpha, \beta)}(z)=\frac{(\alpha+1)_{n}}{n !}{ }_{2} F_{1}(-n, n+\alpha+\beta+1 ; \alpha+1 ;(1-z) / 2)
$$

satisfy the orthogonality relation

$$
\int_{-1}^{1} P_{n}^{(\alpha, \beta)}(x) P_{m}^{(\alpha, \beta)}(x)(1-x)^{\alpha}(1+x)^{\beta} d x=0, \quad n \neq m,
$$

for $n, m=0,1,2, \ldots$, and hence, by [20, Theorem 3.3.1], these polynomials have only real zeros. In our derivation of sums of squares expansions which imply the reality of the zeros of these polynomials we will start out by deriving sums of squares expansions for nonterminating ${ }_{2} F_{1}(a, b ; c ; z)$ hypergeometric series with $|z|<1$ (for convergence).

Let $a, b, c$ be real valued, $c \neq 0,-1,-2, \ldots$, and $|z|<1$. Then formula [6, (51)] gives the expansion

$$
\begin{aligned}
\left|{ }_{2} F_{1}(a, b ; c ; z)\right|^{2} & =\sum_{k=0}^{\infty} \frac{(a)_{k}(b)_{k}(c-a)_{k}(c-b)_{k}}{k !(c)_{k}(c)_{2 k}}\left(x^{2}+y^{2}\right)^{k} \\
& \times{ }_{2} F_{1}\left(a+k, b+k ; c+2 k ; 2 x-x^{2}-y^{2}\right) .
\end{aligned}
$$

Unfortunately, application of the inversion [6, (50)] of [6, (51)] to each of the ${ }_{2} F_{1}\left(a+k, b+k ; c+2 k ; 2 x-x^{2}-y^{2}\right)$ functions on the right side of equation (5.3) just returns one back to the function that is on the left side. Therefore, we use formulas $(44),(45),(50)$ in [6] to obtain, respectively, the expansions

$$
=\sum_{j=0}^{\infty} \frac{(a+k)_{j}(b+k)_{j}}{j !(c+2 k)_{j}}(-1)^{j}\left(x^{2}+y^{2}\right)^{j}{ }_{2} F_{1}(a+k+j, b+k+j ; c+2 k+j ; 2 x),
$$

$$
\begin{aligned}
{ }_{2} F_{1}(a+k+ & j, b+k+j ; c+2 k+j ; 2 x)=\sum_{m=0}^{\infty} \frac{(a+k+j)_{m}(b+k+j)_{m}}{m !(c+2 k+j)_{m}} x^{2 m} \\
& \times{ }_{2} F_{1}\left(a+k+j+m, b+k+j+m ; c+2 k+j+m ; 2 x-x^{2}\right),
\end{aligned}
$$




$$
\begin{aligned}
& \text { (5.6) }{ }_{2} F_{1}\left(a+k+j+m, b+k+j+m ; c+2 k+j+m ; 2 x-x^{2}\right) \\
& =\sum_{n=0}^{\infty} \frac{(a+k+j+m)_{n}(b+k+j+m)_{n}(c-a+k)_{n}(c-b+k)_{n}}{n !(c+2 k+j+m+n-1)_{n}(c+2 k+j+m)_{2 n}}(-1)^{n} x^{2 n} \\
& \times\left({ }_{2} F_{1}(a+k+j+m+n, b+k+j+m+n ; c+2 k+j+m+2 n ; x)\right)^{2},
\end{aligned}
$$

and then substitute these expansions in turn into (5.3), change the order of summation and use the binomial theorem to obtain

$$
\begin{aligned}
& \left|{ }_{2} F_{1}(a, b ; c ; z)\right|^{2}=\sum_{m=0}^{\infty} \sum_{j=0}^{m} \frac{(a)_{m}(b)_{m}(c-a)_{j}(c-b)_{j}}{j !(m-j) !(c)_{m+j}(m+c-1)_{j}}(-1)^{m} x^{2 j} y^{2 m-2 j} \\
& \quad \times{ }_{2} F_{1}\left(-j, m+c-1 ; c ; 1+y^{2} / x^{2}\right)\left({ }_{2} F_{1}(m+a, m+b ; m+j+c ; x)\right)^{2} .
\end{aligned}
$$

Application of (3.7) to the first ${ }_{2} F_{1}$ series on the right side of (5.7) gives

$$
\begin{aligned}
\left.\left.\right|_{2} F_{1}(a, b ; c ; z)\right|^{2} & =\sum_{m=0}^{\infty} \sum_{j=0}^{m} \frac{(a)_{m}(b)_{m}(c-a)_{j}(c-b)_{j}}{j !(m-j) !(c)_{m+j}(m+c-1)_{j}}(-1)^{m+j} y^{2 m} \\
\times{ }_{2} F_{1}(-j, 1 & \left.-m ; c ; 1+x^{2} / y^{2}\right)\left({ }_{2} F_{1}(m+a, m+b ; m+j+c ; x)\right)^{2}
\end{aligned}
$$

which contains (4.14) as a limit case. When $a=-n$ is a negative integer, $b=n+\alpha+\beta+1$ and $c=\alpha+1$, it follows from (5.8) that

$$
\begin{aligned}
& \text { (5.9) }\left|\frac{n !}{(\alpha+1)_{n}} P_{n}^{(\alpha, \beta)}(1-2 z)\right|^{2} \\
&= \sum_{m=0}^{n} \sum_{j=0}^{m} \frac{(-n)_{m}(n+\alpha+\beta+1)_{m}(n+\alpha+1)_{j}(-n-\beta)_{j}}{j !(m-j) !(\alpha+1)_{m+j}(m+\alpha)_{j}}(-1)^{m+j} y^{2 m} \\
& \times{ }_{2} F_{1}\left(-j, 1-m ; \alpha+1 ; 1+x^{2} / y^{2}\right)\left({ }_{2} F_{1}(m-n, m+n+\alpha+\beta+1 ; m+j+\alpha+1 ; x)\right)^{2}
\end{aligned}
$$

which gives a sums of squares proof that the Jacobi polynomials $P_{n}^{(\alpha, \beta)}(z)$ have only real zeros when $\alpha, \beta>-1$ (since the coefficients in (5.9) are then clearly positive) and hence, by continuity, when $\alpha, \beta \geq-1$. The restriction that $\alpha, \beta \geq-1$ cannot be extended to $\alpha, \beta \geq-2$ because $P_{2}^{(\alpha, \beta)}(z)$ has non-real zeros when $\alpha, \beta>-2$ and $\alpha+\beta<-3$.

As in sections 3 and 4 one may repeatedly differentiate (5.7) with respect to $y$ and apply (3.7) to obtain extensions of (4.15), (4.16), etc. But, since the resulting identities are quite lengthly and do not add any additional $(\alpha, \beta)$ 
for which the Jacobi polynomials have only real zeros, we will omit them and only point out that the first two differentiations give identities that yield, in particular, the inequalities

$$
y \frac{\partial}{\partial y}\left|P_{n}^{(\alpha, \beta)}(1-2 z)\right|^{2} \geq \frac{2 n(n+\alpha+\beta+1)_{n}(\alpha+1)_{n}}{n ! n !} y^{2 n}
$$

and

$$
\frac{\partial^{2}}{\partial y^{2}}\left|P_{n}^{(\alpha, \beta)}(1-2 z)\right|^{2} \geq \frac{2 n(2 n-1)(n+\alpha+\beta+1)_{n}(\alpha+1)_{n}}{n ! n !} y^{2 n-2}
$$

when $n \geq 1$ and $\alpha, \beta \geq-1$.

In subsequent papers it will be shown that squares of real valued functions can also be used to prove the reality of the zeros of some non-classical families of orthogonal polynomials, of the cosine transforms

$$
\int_{0}^{\infty} e^{-a \cosh t} \cos z t d t, \quad a>0
$$

and of some other entire functions.

\section{References}

[1] R. Askey and G. Gasper, Positive Jacobi polynomial sums II, Amer. J. Math. 98 (1976), 709-737.

[2] R. Askey and G. Gasper, Inequalities for polynomials, in The Bieberbach Conjecture, Proceedings of the Symposium on the Occasion of the Proof, Surveys and Monographs, No. 21, Amer. Math. Soc., Providence, RI (1986), 7-32.

[3] W.N. Bailey, On the product of two Legendre polynomials with different arguments, Proc. London Math. Soc. (2) 41 (1936), 215-220.

[4] R.P. Boas, Entire Functions, Academic Press, Inc., New York, 1954.

[5] L. de Branges, A proof of the Bieberbach conjecture, Acta Math. 154 (1985), 137-152.

[6] J.L. Burchnall and T.W. Chaundy, Expansions of Appell's double hypergeometric functions, Quart. J. Math. (Oxford) 11 (1940), 249-270.

[7] J.L. Burchnall and T.W. Chaundy, Expansions of Appell's double hypergeometric functions (II), Quart. J. Math. (Oxford) 12 (1941), 112-128. 
[8] L. Carlitz, Some polynomials related to the ultraspherical polynomials, Portugaliae Math. 20 (1961), 127-136.

[9] A. Erdelyi, Higher Transcendental Functions, vols. 1 and 2, McGraw Hill, New York, 1953.

[10] G. Gasper, Positive integrals of Bessel functions, SIAM J. Math. Anal. 6 (1975), 868-881.

[11] G. Gasper, Positivity and special functions, in Theory and Applications of Special Functions, R. Askey, ed., Academic Press, New York (1975), 375-433.

[12] G. Gasper, Positive sums of the classical orthogonal polynomials, SIAM J. Math. Anal. 8 (1977), 423-447.

[13] G. Gasper, A short proof of an inequality used by de Branges in his proof of the Bieberbach, Robertson and Milin conjectures, Complex Variables: Theory Appl. 7 (1986), 45-50.

[14] G. Gasper, $q$-Extensions of Clausen's formula and of the inequalities used by de Branges in his proof of the Bieberbach, Robertson, and Milin conjectures, SIAM J. Math. Anal. 20 (1989), 1019-1034.

[15] G. Gasper, Using symbolic computer algebraic systems to derive formulas involving orthogonal polynomials and other special functions, in Orthogonal Polynomials: Theory and Practice, ed. by P. Nevai, Kluwer Academic Publishers, Boston, 1989, $163-179$.

[16] G. Gasper and M. Rahman, Basic Hypergeometric Series, Cambridge University Press, 1990.

[17] E. Hille, Note on some hypergeometric series of higher order, J. London Math. Soc. 4 (1929), 50-54.

[18] J.L.W.V. Jensen, Recherches sur la théorie des équations, Acta Math. 36 (1913), 181-195.

[19] G. Pólya, Über die algebraisch-funktionentheoretischen Untersuchungen von J. L. W. V. Jensen, Kgl. Danske Videnskabernes Selskab. Math.-Fys. Medd. 7 (17) (1927), pp. 3-33; reprinted in his Collected Papers, Vol. II, pp. 278-308.

[20] G. Szegö, Orthogonal Polynomials, 4th ed., Amer. Math. Soc. Colloq. Publ. 23, Providence, R.I. 1975.

[21] E.C. Titchmarsh, The Theory of the Riemann Zeta-Function, 2nd edition (Revised by D.R. Heath-Brown), Oxford Univ. Press, Oxford and New York, 1986.

[22] R.S. Varga, Scientific Computation on Mathematical Problems and Conjectures, CBMS-NSF Regional Conference Series in Applied Mathematics, SIAM, Philadelphia, 1990.

[23] G.N. Watson, Theory of Bessel Functions, Cambridge Univ. Press, Cambridge and New York, 1944. 


\section{George Gasper}

Department of Mathematics

Northwestern University

Evanston, IL 60208

E-Mail: g-gasper@nwu.edu 